\title{
Correction to: Effect of gymnastic exercises on motor development and neuropsychological characteristics in autistic children
}

\author{
Azadeh Zamani Jam ${ }^{1,2} \cdot$ Rasoul Hemayat Talab ${ }^{3} \cdot$ Mahmoud Sheikh $^{3} \cdot$ Farnaz Torabi $^{4} \cdot$ Forouzan Rafie ${ }^{5}$ (I)
}

Published online: 17 March 2018

(c) Springer-Verlag Italia S.r.l., part of Springer Nature 2018

Correction to: Sport Sciences for Health

$$
\text { https://doi.org/10.1007/s11332-017-0417-y }
$$

Unfortunately, the title of this article has been published incorrectly in the original version. The correct title should read as follows:

Effect of gymnastic exercises on motor development and neuropsychological characteristics in autistic children.

The original article can be found online at https://doi.org/10.1007/ s11332-017-0417-y.

Forouzan Rafie

fr.rafie87@ut.ac.ir

1 University of Tehran, Alborz campus, Tehran, Iran

2 Payame Noor University, Tehran, Iran

3 Faculty of Physical Education and Sport Sciences, University of Tehran, Tehran, Iran

4 Faculty of Physical Education and Sport Sciences, Payame Noor University of Tehran, Tehran, Iran

5 Neuroscience Research Centre, Kerman University of Medical Sciences, Kerman, Iran 